where $\dot{+}$ denotes addition modulo one. Thus $(3)$ is equivalent to requiring that $\Theta_{1} \dot{+} \Theta_{2}$ and $\Theta_{1}$ have the same distribution. If we set

$$
\phi(n)=E\left\{e^{2 \pi i n \theta_{1}}\right\}=\int_{0}^{1} e^{2 \pi i n \theta_{1}} d F_{\Theta_{1}}\left(\theta_{1}\right),
$$

then (3) and the independence of $\Theta_{1}, \Theta_{2}$ imply

$$
\phi(n)=E\left\{e^{2 \pi i n\left(\boldsymbol{\theta}_{1}+\boldsymbol{\theta}_{2}\right)}\right\}=E\left\{e^{2 \pi i n\left(\boldsymbol{\theta}_{1}+\boldsymbol{\theta}_{2}\right)}\right\}=\phi^{2}(n)
$$

so that $\phi(n)=0$ or 1 . Certainly $\phi(0)=1$. There are two cases to be examined.

Case 1. $\phi(n)=0$ for all $n \neq 0$.

It follows from the uniqueness theorem for Fourier-Stieltjes series that $d F_{\boldsymbol{\theta}_{1}}\left(d \theta_{1}\right)=d \theta_{1}$ and hence $\operatorname{Pr}\left(M_{1} \leqq x\right)=F_{\infty}(x)$.

Case 2. $\phi(n)=1$ for some $n \neq 0$.

Let $m$ be the smallest positive integer such that $\phi(m)=1$. Then

$$
0=\int_{0}^{1}\left(1-e^{2 \pi i m \theta_{1}}\right) d F_{\Theta_{1}}\left(\theta_{1}\right)=\int_{0}^{1}\left(1-\cos 2 \pi m \theta_{1}\right) d F_{\Theta_{1}}\left(\theta_{1}\right) .
$$

It follows that $F_{\boldsymbol{\theta}_{1}}$ is a 'step function' with points of discontinuity at $\theta_{k}=k / m$ $(k=1,2, \cdots, m)$ and, hence, $\phi(n+m)=\phi(n)(n=0, \pm 1, \pm 2, \cdots)$. We assert that $\phi(n)=1$ if and only if $n=k m$ for some integer $k$; for if $\phi(n)=1$ with $k m<n<(k+1) m$ then $\phi(n-k m)=\phi(n)=1$ while $0<n-k m<m$ contradicting the minimality of $m$. The uniqueness theorem for Fourier-Stieltjes series now shows that $\operatorname{Pr}\left(M_{1} \leqq x\right)=F_{m}(x)$.

I should like to acknowledge with thanks several suggestions made by $\mathrm{Mr}$. Benjamin Weiss.

Thomas J. Watson Research Center

International Business Machines Corporation

Yorktown Heights, New York

\title{
New Primes of the Form $n^{4}+1$
}

\section{By A. Gloden}

This note presents some results of a continuation of the author's systematic factorization of integers of the form $n^{4}+1$ [1].

An electronic computer at l'Institut Blaise Pascal in Paris has been used to find solutions of the congruence $x^{4}+1 \equiv 0(\bmod p)$ for all primes of the form $8 k+1$ in the interval $10^{6}<p<4 \cdot 10^{6}$, thereby extending the previous range of such tables listed in [1].

With the aid of these tables, the complete factorization of $n^{4}+1$ has now been effected for all even values of $n$ less than 2040 and for all odd values less than 2397 .

Thus, the primality of $\frac{1}{2}\left(n^{4}+1\right)$ has been established for the following 116 values of $n$ :

Received February 25, 1963. Revised August 2, 1963. 


$\begin{array}{llllllll}1007 & 1163 & 1401 & 1655 & 1853 & 2051 & 2205 & 2349 \\ 1013 & 1173 & 1441 & 1683 & 1865 & 2061 & 2209 & 2363 \\ 1015 & 1183 & 1457 & 1687 & 1905 & 2069 & 2215 & 2369 \\ 1019 & 1253 & 1463 & 1737 & 1909 & 2071 & 2223 & 2373 \\ 1041 & 1259 & 1483 & 1745 & 1915 & 2073 & 2245 & \\ 1047 & 1269 & 1485 & 1751 & 1935 & 2079 & 2247 & \\ 1049 & 1275 & 1493 & 1755 & 1945 & 2097 & 2255 & \\ 1053 & 1305 & 1527 & 1757 & 1967 & 2125 & 2261 & \\ 1057 & 1327 & 1529 & 1765 & 1977 & 2131 & 2279 & \\ 1071 & 1333 & 1533 & 1789 & 1985 & 2141 & 2283 & \\ 1087 & 1353 & 1547 & 1809 & 2001 & 2143 & 2305 & \\ 1101 & 1355 & 1557 & 1813 & 2007 & 2145 & 2311 & \\ 1119 & 1371 & 1567 & 1823 & 2011 & 2149 & 2315 & \\ 1123 & 1381 & 1569 & 1829 & 2013 & 2163 & 2333 & \\ 1125 & 1383 & 1571 & 1841 & 2037 & 2175 & 2341 & \\ 1135 & 1389 & 1635 & 1849 & 2039 & 2193 & 2343 & \end{array}$

Similarly, corresponding to the following 94 values of $n$, the integer $n^{4}+1$ has been shown to be prime:

$\begin{array}{llllllll}1038 & 1170 & 1322 & 1472 & 1598 & 1688 & 1824 & 1942 \\ 1042 & 1180 & 1330 & 1486 & 1610 & 1700 & 1836 & 1944 \\ 1072 & 1200 & 1344 & 1496 & 1612 & 1706 & 1850 & 1948 \\ 1076 & 1202 & 1382 & 1536 & 1618 & 1710 & 1854 & 1952 \\ 1088 & 1218 & 1388 & 1540 & 1622 & 1718 & 1864 & 1956 \\ 1126 & 1236 & 1404 & 1542 & 1638 & 1722 & 1870 & 1962 \\ 1132 & 1238 & 1406 & 1552 & 1644 & 1738 & 1892 & 1972 \\ 1136 & 1246 & 1428 & 1554 & 1646 & 1754 & 1910 & 1978 \\ 1142 & 1252 & 1434 & 1558 & 1650 & 1772 & 1916 & 1986 \\ 1144 & 1270 & 1442 & 1568 & 1652 & 1788 & 1926 & 1994 \\ 1150 & 1280 & 1446 & 1586 & 1666 & 1806 & 1932 & \\ 1152 & 1302 & 1458 & 1594 & 1680 & 1820 & 1934 & \end{array}$

11 Rue Jean Jaurès

Luxembourg

1. A. Gloden, "Additions to Cunningham's Factor Table of $n^{4}+1$," Math. Comp., v. 16, 1962, p. 239-241.

\section{Some Additional Factorizations of $2^{n} \pm 1$}

\section{By K. R. Isemonger}

Herein are set forth some details of three new factorizations of integers of the form $2^{n} \pm 1$.

The first of these is the complete factorization of $2^{119}-1$, which possesses as algebraic factors the Mersenne primes $2^{7}-1=127$ and $2^{17}-1=131071$. The quotient is known to be divisible by 239 and 20231. There then remains the factorization of the integer

$$
N=8257410955834335790279,
$$

which was proved composite by E. Gabard of Poitiers, France.

Received November 29, 1963. Revised May 15, 1964. 